\title{
Impact of leptoquarks in semileptonic $B$ decays
}

\author{
Suchismita Sahoo* $\dagger$ \\ School of Physics, University of Hyderabad, \\ Hyderabad-500046, India \\ E-mail: suchismita@uohyd.ac.in
}

\section{Rukmani Mohanta}

School of Physics, University of Hyderabad,

Hyderabad-500046, India

E-mail: rmsp@uohyd.ernet.in

\section{Anjan K. Giri}

Physics Department, IIT Hyderabad,

Kandi - 502285, India

E-mail: giria@ith.ac.in

We study the impact of vector leptoquarks on the recent anomalies in semileptonic $B$ meson decays such as $R_{K}$ and $R_{D^{(*)}}$ etc. We constrain the leptoquark couplings by using the existing data on the branching ratios of $B_{s} \rightarrow l^{+} l^{-}$and $\bar{B} \rightarrow X_{s} l^{+} l^{-}$processes, where $l=e, \mu, \tau$. We estimate the branching ratios of $\bar{B} \rightarrow D^{(*)} l \bar{v}_{l}$ processes using the constrained leptoquark parameter space. We also investigate the possibility of simultaneous explanation of $R_{D^{(*)}}, R_{K}$ anomalies in this model.

9th International Workshop on the CKM Unitarity Triangle

28 November - 3 December 2016

Tata Institute for Fundamental Research (TIFR), Mumbai, India

\footnotetext{
* Speaker.

${ }^{\dagger}$ The authors would like to thank Science and Engineering Research Board (SERB), Government of India for financial support through grant No. SB/S2/HEP-017/2013.
} 


\section{Introduction}

Recently both $\mathrm{BaBar}[1]$ and Belle [2] experiments have measured the ratio of branching fractions of $\bar{B} \rightarrow D \tau \bar{v}_{\tau}$ over $\bar{B} \rightarrow D l \bar{v}_{l}$ decays, where $l=e, \mu$ and the present experimental average [3] is

$$
R_{D}=\frac{\operatorname{Br}\left(\bar{B} \rightarrow D \tau \bar{v}_{\tau}\right)}{\operatorname{Br}\left(\bar{B} \rightarrow D l \bar{v}_{l}\right)}=0.397 \pm 0.040 \pm 0.028
$$

which has $1.9 \sigma$ deviation from its standard model (SM) prediction $R_{D}^{\mathrm{SM}}=0.300 \pm 0.008$ [4]. Besides, both the $B$ factories and LHCb [5] have reported 3.3 $\sigma$ discrepancy [3] in the measurement of $R_{D^{*}}$

$$
R_{D^{*}}=\frac{\operatorname{Br}\left(\bar{B} \rightarrow D^{*} \tau \bar{v}_{\tau}\right)}{\operatorname{Br}\left(\bar{B} \rightarrow D^{*} l \bar{v}_{l}\right)}=0.316 \pm 0.016 \pm 0.010
$$

from its SM result $R_{D^{*}}^{\mathrm{SM}}=0.252 \pm 0.003$ [6]. Analogously another interesting observable is the lepton nonuniversality (LNU) parameter $\left(R_{K}\right)$ in $B^{+} \rightarrow K^{+} l^{+} l^{-}$process, which has recently been measured at $\mathrm{LHCb}$, with value [7],

$$
R_{K}=\frac{\operatorname{Br}\left(B^{+} \rightarrow K^{+} \mu^{+} \mu^{-}\right)}{\operatorname{Br}\left(B^{+} \rightarrow K^{+} e^{+} e^{-}\right)}=0.745_{-0.074}^{+0.090} \pm 0.036
$$

and corresponds to a $2.6 \sigma$ deviation from its SM value $R_{K}=1.0003 \pm 0.0001$ [8] in the dilepton invariant mass squared bin $\left(1 \leq q^{2} \leq 6\right) \mathrm{GeV}^{2}$.

In this paper, we investigate the semileptonic $\bar{B} \rightarrow D^{(*)} l \bar{v}_{l}$ decay processes, mediated by the FCNC transitions $b \rightarrow c l \bar{v}_{l}$ in the vector leptoquark (LQ) model. We compute the branching ratios of $\bar{B} \rightarrow D^{(*)} l \bar{v}_{l}$ modes in this model. A simultaneous study of the LNU parameters $R_{D^{(*)}}$ and $R_{K^{(*)}}$ is the main objective of this work. LQs are color triplet hypothetical bosonic particles, which allow quarks and leptons to interact simultaneously and carry both baryon and lepton numbers. They can have spin 0 (scalar) or spin 1 (vector) and are encountered in various extensions of the SM, such as technicolor model [9], GUT theories [10, 11], Pati-Salam models [11, 12] and the quark and lepton composite model [13].

The outline of the paper is follows. In section 2, we discuss the effective Hamiltonians involving $b \rightarrow c l \bar{v}_{l}$ and $b \rightarrow s l l$ transitions in the SM. We also present the new physics contribution due to the vector LQ exchange. The constraint on LQ parameter space by using the experimental limit on the branching ratios of the $B_{s} \rightarrow l^{+} l^{-}$and $\bar{B} \rightarrow X_{s} l^{+} l^{-}$decays and the numerical analysis for $\bar{B} \rightarrow D^{(*)} l \bar{v}_{l}$ processes are presented in section 3. Section 4 contains the conclusion.

\section{Effective Hamiltonian and the new physics contribution from vector LQ exchange}

In the SM, the effective Hamiltonian describing the processes mediated by the $b \rightarrow c l \bar{v}_{l}$ transition is given by [14]

$$
\mathscr{H}_{\mathrm{eff}}=\frac{4 G_{F}}{\sqrt{2}} V_{c b}\left[\left(\delta_{l \tau}+C_{V_{1}}^{l}\right)\left(\bar{c}_{L} \gamma^{\mu} b_{L}\right)\left(\bar{\tau}_{L} \gamma_{\mu} v_{l L}\right)\right]
$$


where $G_{F}$ is the Fermi constant, $V_{c b}$ is the CKM matrix element and $q_{L(R)}=L(R) q$ are the chiral quark fields with $L(R)=\left(1 \mp \gamma_{5}\right) / 2$ as the projection operators. The Wilson coefficient $C_{V_{1}}^{l}$ is zero in the SM and can only be generated by the new physics model.

Similarly, the effective Hamiltonian for $b \rightarrow s l^{+} l^{-}$process in the SM is given by

$$
\mathscr{H}_{\text {eff }}=-\frac{4 G_{F}}{\sqrt{2}} V_{t b} V_{t s}^{*} \sum_{i=1}^{10} C_{i}(\mu) \mathscr{O}_{i}(\mu)
$$

where $\mathscr{O}_{i}$ 's are the six dimensional operators and $C_{i}$ 's are the corresponding Wilson coefficients.

Models with vector LQs can modify the SM effective Hamiltonian $(2.1,2.2)$ due to the additional contributions arising from the LQ exchange and give measurable deviations from the SM predictions in the beauty sector. Here we consider $U_{3}(3,3,2 / 3)$ vector LQ multiplet that is invariant under the $S U(3)_{c} \times S U(2)_{L} \times U(1)_{Y}$ gauge group of the SM and does not allow proton to decay. The interaction Lagrangian for $U_{3}$ LQ with the SM fermions is given by [14]

$$
\mathscr{L}^{\mathrm{LQ}}=h_{3 L}^{i j} \bar{Q}_{i L} \boldsymbol{\sigma} \gamma^{\mu} L_{j L} \mathbf{U}_{3 \mu}
$$

where $Q(L)$ is the left handed quark (lepton) doublets, $h_{3 L}^{i j}$ are the LQ couplings and $\boldsymbol{\sigma}$ represents the Pauli matrices. After performing the Fierz transformation and comparing with Eqn. (2.1), we obtain an additional Wilson coefficient as

$$
C_{V_{1}}^{l}=-\frac{1}{2 \sqrt{2} G_{F} V_{c b}} \sum_{k=1}^{3} V_{k 3} \frac{h_{3 L}^{2 l} h_{3 L}^{k 3^{*}}}{M_{U_{3}^{2 / 3}}^{2}}
$$

Similarly, the comparison of Eqn. (2.3) after Fierz transformation, with the SM effective Hamiltonian (2.2), one can obtain new Wilson coefficients $C_{9,10}^{N P}$

$$
C_{9}^{\mathrm{NP}}=-C_{10}^{\mathrm{NP}}=\frac{\pi}{\sqrt{2} G_{F} V_{t b} V_{t s}^{*} \alpha} \frac{h_{3 L}^{n i} h_{3 L}^{m j^{*}}}{M_{U_{3}^{2 / 3}}^{2}}
$$

\section{Constraint on LQ couplings and numerical analysis}

After knowing the new physics contribution to the SM, we now proceed to constrain the new parameter space by using the experimental limit on the branching ratios of $B_{s} \rightarrow l^{+} l^{-}$and $\bar{B} \rightarrow$ $X_{s} l^{+} l^{-}$processes. Including LQ model, the branching ratio of $B_{s} \rightarrow l^{+} l^{-}$process is given by

$$
\operatorname{Br}\left(B_{s} \rightarrow l^{+} l^{-}\right)=\frac{G_{F}^{2}}{16 \pi^{3}} \tau_{B_{s}} \alpha^{2} f_{B_{s}}^{2}\left|C_{10}^{\mathrm{SM}}\right|^{2} M_{B_{s}} m_{l}^{2}\left|V_{t b} V_{t s}^{*}\right|^{2} \sqrt{1-\frac{4 m_{l}^{2}}{M_{B_{s}}^{2}}}\left|1+\frac{C_{10}^{\mathrm{NP}}}{C_{10}^{\mathrm{SM}}}\right|^{2}
$$

Now comparing the theoretical value of branching ratio with the $1 \sigma$ range of the experimental data, the constraint on the real and imaginary part of the LQ couplings are given in Table 1.

The branching ratio of $\bar{B} \rightarrow X_{s} l l$ process in the LQ model is given by

$$
\begin{aligned}
\left(\frac{d \mathrm{Br}}{d s_{1}}\right)_{\mathrm{LQ}} & =B_{0}\left[\frac { 1 6 } { 3 } ( 1 - s _ { 1 } ) ^ { 2 } ( 1 + 2 s _ { 1 } ) \left[\operatorname { R e } \left(C_{9}^{\mathrm{eff}} C_{9}^{\mathrm{NP}}+\operatorname{Re}\left(C_{10} C_{10}^{\mathrm{NP} *}\right]\right.\right.\right. \\
& \left.+\frac{8}{3}\left(1-s_{1}\right)^{2}\left(1+2 s_{1}\right)\left[\left|C_{9}^{\mathrm{NP}}\right|^{2}+\left|C_{10}^{\mathrm{NP}}\right|^{2}\right]+32\left(1-s_{1}\right)^{2} \operatorname{Re}\left(C_{7} C_{10}^{\mathrm{NP}}\right)\right]
\end{aligned}
$$


where $s_{1}=q^{2} / m_{b}^{2}$ and $B_{0}$ can be found in [15]. The allowed region of corresponding LQ couplings which are compatible with the $1 \sigma$ range of the experimental result in low $q^{2}$ are given in Table 1 .

Using the constrained LQ couplings from Table 1 and Eqns. (2.4, 2.5), we compute the bound on new Wilson coefficients $C_{V_{1}}^{l}, C_{9,10}^{\mathrm{NP}}$. In Table II, we present the predicted values of branching ratios of $B \rightarrow D^{(*)} l v_{l}$ processes in both the SM and $U_{3}$ LQ model. In Fig. 1, we show the $q^{2}$ variation of LNU parameters, $R_{D}\left(q^{2}\right)$ (left panel) and $R_{D^{*}}\left(q^{2}\right)$ (right panel). The plots for $R_{K}\left(q^{2}\right)$ (left panel) and $R_{K^{*}}\left(q^{2}\right)$ (right panel) parameters in low $q^{2}$ region are shown in Fig. 2. The corresponding numerical values of LNU parameters are given in Table 2.

Table 1: Constraints on the real and imaginary parts of the leptoquark couplings (for $M_{\mathrm{LQ}}=1 \mathrm{TeV}$ ) from $B_{s} \rightarrow l^{+} l^{-}$and $\bar{B} \rightarrow X_{s} l l$ processes [15]

\begin{tabular}{|c|c|c|c|}
\hline Deacy process & Leptoquark couplings & Real part & Imaginary Part \\
\hline & $h_{3 L}^{21} h_{3 L}^{31^{*}}$ & $-13.0 \rightarrow 13.0$ & $-13 \rightarrow 13$ \\
$B_{S} \rightarrow l^{+} l^{-}$ & $h_{3 L}^{22} h_{3 L}^{32^{*}}$ & $-0.016 \rightarrow 0.0$ & $-0.008 \rightarrow 0.008$ \\
& $h_{3 L}^{23} h_{3 L}^{33^{*}}$ & $-0.4 \rightarrow 0.4$ & $-0.4 \rightarrow 0.4$ \\
\hline $\bar{B} \rightarrow X_{s} l^{+} l^{-}$ & $h_{3 L}^{21} h_{3 L}^{31^{*}}$ & $-0.01 \rightarrow 0.01$ & $-0.01 \rightarrow 0.01$ \\
& $h_{3 L}^{22} h_{3 L}^{32^{*}}$ & $-0.008 \rightarrow 0.008$ & $-0.008 \rightarrow 0.008$ \\
\hline
\end{tabular}
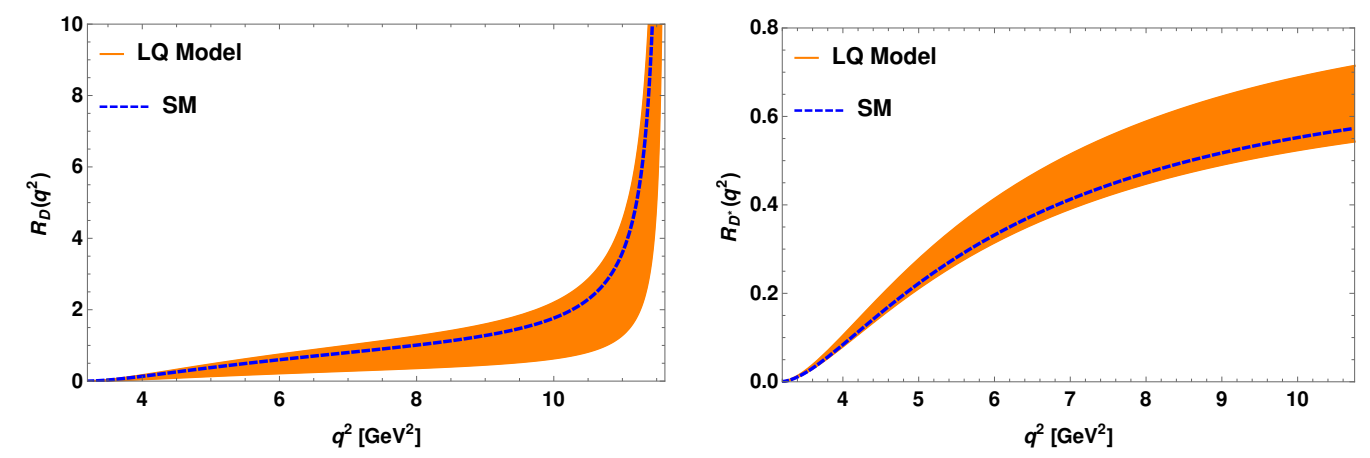

Figure 1: The $q^{2}$ variation of $R_{D}\left(q^{2}\right)$ (left panel) and $R_{D^{*}}\left(q^{2}\right)$ (right panel) parameters in LQ model.

\section{Conclusions}

In this work we have studied the semileptonic $\bar{B} \rightarrow D^{(*)} l \bar{v}_{l}$ decays and the LNU parameters such as $R_{D^{(*)}}$ and $R_{K^{(*)}}$ in the $U_{3}(3,3,2 / 3)$ vector LQ model. The LQ parameter space is constrained by using the branching ratios of rare $B_{s} \rightarrow l^{+} l^{-}$and $\bar{B} \rightarrow X_{s} l^{+} l^{-}$decay processes. We computed the branching ratios and lepton nonuniversality in $\bar{B} \rightarrow D^{(*)} l \bar{v}_{l}$ processes in the $U_{3}$ vector LQ model. The anomaly in $R_{K}$ observable is also studied. We found that the observed anomalies can be accommodated in this model.

\section{References}

[1] J. P. Lees et al., [BaBar Collaboration], Phys. Rev. Lett. 109, 101802 (2012); J. P. Lees et al., [BaBar Collaboration], Phys. Rev. D 88, 072012 (2013). 
Table 2: The predicted values of branching ratios of $\bar{B} \rightarrow D^{(*)} l \bar{v}_{l}$ processes and the $R_{D^{(*)}}, R_{K^{(*)}}$ anomalies in vector leptoquark model $[15,16]$

\begin{tabular}{|c|c|c|}
\hline Observables & SM predictions & Values in LQ Model \\
\hline $\operatorname{Br}\left(\bar{B} \rightarrow D l \bar{v}_{l}\right)$ & $(2.18 \pm 0.13) \times 10^{-2}$ & $(2.13-2.25) \times 10^{-2}$ \\
$\operatorname{Br}\left(\bar{B} \rightarrow D \tau \bar{v}_{l}\right)$ & $(6.75 \pm 0.08) \times 10^{-3}$ & $(2.48-8.2) \times 10^{-3}$ \\
$\operatorname{Br}\left(\bar{B} \rightarrow D^{*} l \bar{v}_{l}\right)$ & $(5.18 \pm 0.31) \times 10^{-2}$ & $(5.04-5.32) \times 10^{-2}$ \\
$\operatorname{Br}\left(\bar{B} \rightarrow D^{*} \tau \bar{v}_{l}\right)$ & $(1.33 \pm 0.14) \times 10^{-2}$ & $(1.3-1.6) \times 10^{-2}$ \\
$R_{D}$ & 0.31 & $0.11-0.386$ \\
$R_{D^{*}}$ & 0.26 & $0.243-0.32$ \\
$R_{K_{q^{2}} \in[1,6]}$ & 1.006 & $0.75-1.006$ \\
$R_{K^{*} q^{2} \in[1,6]}$ & 0.996 & $0.725-0.996$ \\
\hline
\end{tabular}
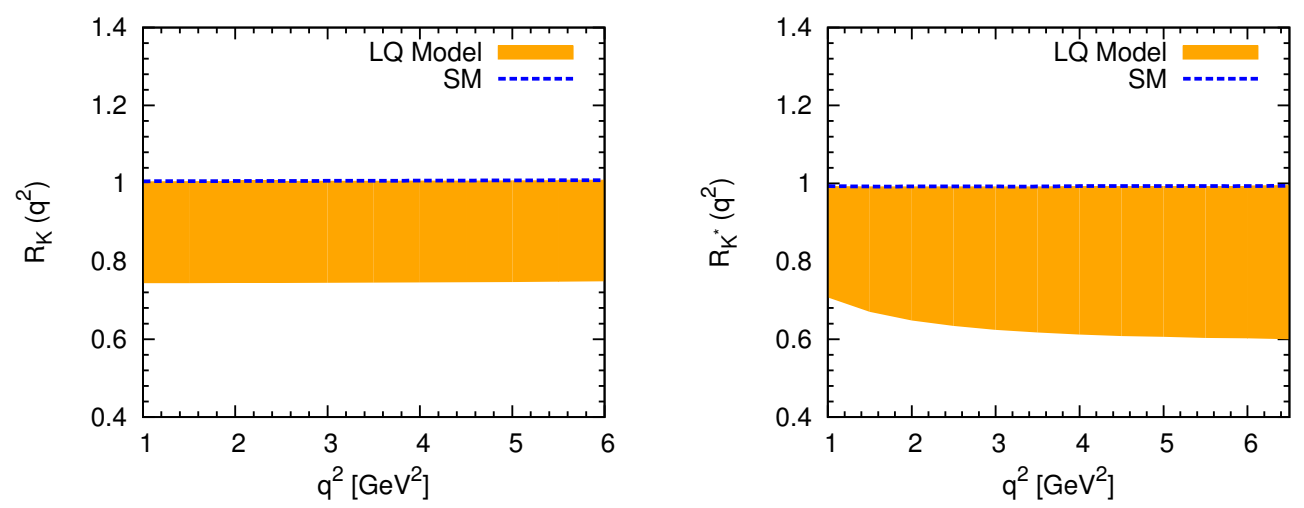

Figure 2: The plot for $R_{K}\left(q^{2}\right)$ (left panel) and $R_{K^{*}}\left(q^{2}\right)$ (right panel) in the low $q^{2}$ region in LQ model.

[2] M. Huschle et al., [Belle Collaboration], Phys. Rev. D 92, 072014 (2015); A. Abdesselam et al., [Belle Collaboration], [arXiv:1603.06711].

[3] Heavy Flavour Averaging Group, http://www.slac.stanford.edu/xorg/hfag/semi/winter16/ winter16_dtaunu.html.

[4] H. Na et al., [HPQCD Collaboration], Phys. Rev. D 92, 054410 (2015).

[5] R. Aaij et al. [LHCb Collaboration], Phys. Rev. Lett. 115, 111803 (2015) Erratum: Phys. Rev. Lett. 115, 159901 (2015).

[6] S. Fajfer, J. F. Kamenik, and I. Nisandzic, Phys. Rev. D 85, 094025 (2012).

[7] R. Aaij et al., [LHCb Collaboration], Phys. Rev. Lett. 113, 151601 (2014).

[8] C. Bobeth, G. Hiller, and G. Piranishvili, JHEP 12, 040 (2007).

[9] B. Schrempp, and F. Shrempp, Phys. Lett. B 153, 101 (1985); B. Gripaios, JHEP 1002, 045 (2010) [arXiv:0910.1789].

[10] H. Georgi, and S. L. Glashow, Phys. Rev. Lett. 32, 438 (1974); H. Fritzsch and P. Minkowski, Ann. Phys. 93, 193 (1975); P. Langacker, Phys. Rep. 72, 185 (1981). 
[11] J. C. Pati, and A. Salam, Phys. Rev. D 10, 275 (1974).

[12] J.C. Pati, and A. Salam, Phys. Rev. D 8, 1240(1973); Phys. Rev. Lett. 31, 661 (1973); O. Shenkar, Nucl. Phys. B 206, 253 (1982); O. Shenkar, Nucl. Phys. B 204, 375 (1982).

[13] D. B. Kaplan, Nucl. Phys. B 365, 259 (1991).

[14] Y. Sakaki, R. Watanabe, M. Tanaka, and A. Tayduganov, Phys. Rev. D 88, 094012 (2013).

[15] S. Sahoo, R. Mohanta, and A. Giri, [arXiv:1609.04367].

[16] A. Giri, R. Mohanta, and S. Sahoo, J.Phys.Conf.Ser. 770, 012031 (2016). 